\title{
Leveraging the expertise of the urban STEM student in developing an effective LA Program: LA and instructor partnerships
}

\author{
Mel S. Sabella, Andrea G. Van Duzor, and Felicia Davenport \\ Department of Chemistry, Physics, and Engineering Studies, Chicago State University, \\ 9501 S. King Drive, Chicago, IL, 60628
}

\begin{abstract}
A key component to the success of the Learning Assistant (LA) Model is the relationship that forms between LAs and faculty members. These relationships can enhance the effectiveness of the model by leveraging the expertise of the LAs and placing LAs in leadership roles where they can co-think and codesign activities and lessons with faculty, as well as provide insights to faculty about the students in the class and the learning environment. Interviews with LAs and faculty members, in addition to video from weekly preparation sessions, illustrate the different types of partnerships that can evolve between LAs and faculty and help us understand the roles different factors play in these partnerships. We contrast three different types of partnerships between LAs and faculty that exist along a continuum: mentor-mentee, faculty-driven collaboration, and collaborative. This data highlights the importance and the benefits of being attentive to these partnerships in developing a robust and effective LA Program.
\end{abstract}

\section{INTRODUCTION}

The Learning Assistant (LA) Model places undergraduate students, LAs, into facilitator roles in the classroom [1]. This model, developed at the University of Colorado-Boulder, provides LAs with three essential elements: a pedagogy course, weekly meetings with faculty mentors, and in class time supporting their peers' learning, with continuous reflection on the teaching and learning of the subject throughout [2]. These elements provide substantial support to LAs and unique opportunities for collaboration and partnerships between undergraduates (LAs) and faculty. LA Programs, at institutions using the LA model, have been shown to create positive shifts in overall LA attitudes about science, personal interest, and content understanding [3]. In addition, they can serve to facilitate students' growth of identity as scientists and inclusion in the scientific community of practice [4]. A critical component of the LA model is that it can be transformative not only to the LAs who participate, but that it can also impact faculty, as they work toward transforming their courses to be able to utilize LAs.

The LA Program at Chicago State University (CSU) has grown from three students in a single discipline to nineteen students in five disciplines. At the international level, the LA Model has seen great growth in recent years, with over seventy institutions running some form of an LA Program to support students, mainly in STEM. Some of these programs are well established while others are emerging [1]. As LA programs proliferate it is important to understand the structure of LA-faculty partnerships and to elucidate student-faculty co-development of instructional environments that build on the expertise of both the LAs and the faculty. This study examines LA-faculty partnerships at CSU, where LAs can leverage their understanding of both learners and the local community, to impact the instructional environment. Three types of partnerships are explored: mentor-mentee, faculty-driven collaboration, and collaborative.

\section{BACKGROUND}

\section{A. Motivation for this work in the urban context}

While all LA Programs incorporate the elements described in the introduction, the specifics of individual programs varies, based on local characteristics, needs, strengths, and resources. For example, at CSU, because of the strong ties to local high school teachers and to a local community, the CSU pedagogy course is led by two Chicago area high school teachers and incorporates a teaching episode that is implemented in either a high school or a college setting $[5,6]$. CSU primarily serves the population from the Southside of Chicago and this collective local knowledge is leveraged in our LA Program. While the CSU faculty are heterogeneous, coming from all over the world with diverse experiences, the majority of our students come from nearby communities and have many shared experiences, cultural resources, and knowledge of the CSU community that our faculty often do not have [7].

In a diverse, yet, segregated city like Chicago, significant cultural differences exist from neighborhood to neighborhood [8]. It is therefore extremely important for us to create spaces for student input, leadership, and voice in our science program. Without this, there is the potential for instructors to fall into "commonly accepted notions on science education [that] abandon communal approaches ... [and engage in] practices that are far removed from 
students' ways of knowing [9]." The LA Model can create mechanisms to provide this space and create opportunities for partnerships to develop between LAs and faculty, where each is contributing to the academic environment.

Partnerships between LAs and faculty can take on many of the characteristics of communities of practice where the members share a common concern and together learn how to address that concern in better ways [10]. A CSU Physics LA expressed this, in a written reflection, stating, "I feel that it is most important for a partnership to form if both the LA and the faculty member have the same goal in mind - to help the students." While LAs can help instructors implement the type of learning environments that instructors strive for, they can also co-create these learning environments with instructors. This is especially important at institutions where the culture of the students differs from the culture of the instructor. In urban settings, "the enactment of communal practices that reclaim ownership of science ... expands agency in urban science classes for historically marginalized students [9]."

\section{B. Weekly preparation sessions}

All three elements of the LA Model play roles in developing partnerships. However, the weekly preparation session, in which an LA meets with the faculty member, typically plays the most important role because of the concentrated time of LA-faculty interaction and focus on the specific course and its students. The weekly preparation portion of the model can vary greatly from institution to institution. While a small LA Program, like CSU $(\sim 20$ LAs), may include one-on-one weekly meetings where the LA talks directly to the instructor of the course, large LA Programs, like the University of Colorado ( 300 LAs), might have twenty LAs and TAs in a weekly meeting with a faculty member, who may not be teaching the course. There is also variability within institutions from faculty to faculty. These meetings depend on the LA and faculty preparation in, and views on science content, pedagogy, and partnership, as well as time constraints for meeting.

While weekly meetings may focus simply on content preparation, they have the potential to do much more. They can share features of cogenerative dialogues, which Tobin has successfully used in urban schools to foster engagement and transform learning environments. In cogenerative dialogues a small number of students and the co-teachers review evidence from a recent class and "cogenerate" collective resolutions regarding new rules for the class, changes in teacher and student roles, and responsibility for accomplishing changes [11]. These cogenerative dialogues create new community spaces where students and instructors both contribute. Emdin describes the importance of membership in a community for students, both in and out of the classroom, noting that membership and responsibility can make our students feel as though they are valued citizens of a community [12]. Faculty willing to see LAs as part of the teaching-learning community can not only increase LAs' sense of science identity but also foster meaningful changes to their courses.

\section{CONTEXT OF THE STUDY}

Data for this study comes from one-on-one interviews with five LAs and seven faculty from the STEM Programs at CSU. All faculty who were interviewed participated in CSU's LA Program and range from first year teachers to those near retirement and were in adjunct or tenured/tenure track positions. LA interviews included traditional and non-traditional students, from first year students through seniors. We describe the specific status only when pertinent to the analysis. Those interviewed were involved in weekly meetings which spanned from no weekly meeting with no LA input, to weekly meetings that lasted over an hour with significant LA input. To supplement the one on one interviews, we present a more in depth look at a specific partnership between a chemistry faculty member, Dr. Anderson, and her LA, Maya, in an upper-level biochemistry course. Their partnership provides an example of the level of collaboration possible and is chosen as an illustrative case study. Videos of two weekly preparation sessions, from the middle of the semester, and a group interview, with the both of them, serve as the data source for this piece of the study. IRB protocols were followed and all names presented are pseudonyms.

\section{LA-FACULTY RELATIONSHIPS}

Relationships between LAs and faculty evolve in a number of ways with varying degrees of contribution and collaboration. The categories of mentor-mentee partnership, faculty driven collaboration, and collaborative partnership characterize these partnerships.

\section{A. The mentor-mentee partnership}

In mentor-mentee partnerships the relationship is onedirectional with limited LA input on classroom dynamics. For example, one faculty member, while desiring more collaboration, described his current relationship as a mentor-mentee relationship because his LAs needed to work on their own content knowledge and take responsibility for being leaders within his courses by having better time management. He stated, "I really ... see it as a mentor-mentee relationship ... what it's come down to is just the content ... I would like ...[the weekly meetings] to be more [about] teaching ..." His weekly meetings consisted primarily of reviewing content and describing the next laboratory, rather than reflection on effective teaching.

\section{B. The two types of collaborative partnerships}

In faculty driven collaboration, faculty elicit feedback and insights from LAs, but still guide how LAs will be 
involved in the course and LAs do not co-design instructional materials. For instance, one faculty member commented, "I tended to have the activities that I wanted them to do and that's what we did, so I didn't often say, 'What do you think we should be doing?' ... I kept pretty close control of the curriculum." In a collaborative partnership, faculty elicit feedback and insights from LAs, and then, together, determine a new activity or way to present material that is then implemented. One faculty member described the collaboration through a problem solving example saying, "it's a lot of back-and-forth. ... [the LA will] say ...'oh, well nobody really liked this problem or I didn't really think that it helped ... [them] understand,' and so then we'll come up with another." Not only did the LA provide feedback on an issue, the LA and the faculty member worked together to create a new problem.

The type of relationship formed is dependent on both the faculty's and the LA's views of their role in the LA model as well as their desires and capacities. While recognizing the difference in power between the LA and faculty, collaborative partnerships require faculty to be willing to share control. One physics faculty expressed this idea stating, "I mean - I'm sort of the boss, but I don't feel like I'm a boss - I feel like the LA has, you know, ideas ... especially good LAs have ideas of their own and - really as a partner - certainly the case with ... the LA that I have now ... you know, we try to work together to figure out what's going to be best for the students." The effectiveness of collaborative partnerships is also dependent on the individual LA. The same faculty member noted that when the content and/or pedagogical preparation of the LA are not as strong, it was difficult for the relationship to be a true collaborative partnership. She stated that "there are some Learning Assistants ... who needed more guidance and in that case I felt ... more like I was the boss and ... they were doing as I said, but I don't like that as much."

When faculty are open to feedback, and LAs are willing and able to take more responsibility, then collaborative partnerships can result, where LA contributions are more substantive. While in each type of partnership, LAs and faculty may share a common goal of supporting students and this may lead to co-membership in a teaching-learning community, the collaborative partnership provides opportunities for the LA and faculty to negotiate, as a team, what this support may look like and allow better alignment of shared goals. During these interactions, as LAs negotiate their space in the teaching-learning community, by bringing in their own resources and knowledge, they develop identity as science people and as teachers. Many of the LAs we interviewed sought to be a part of collaborative partnerships and recognized the importance of their input and membership in the LA Program, specifically describing how they contribute to this community of practice. One LA mentioned that "I know some professors might think ... it's not beneficial ... because they have a certain teaching style ... but I think that if we all work as one ... - as a professor, your kinda confused about why students don't understand ... but if you had your LA there, then you'd be able to ask your LA ... then you guys can work together ..."

\section{The partnership between Maya and Anderson}

The partnership between Maya and Anderson most resembles a collaborative partnership. Maya describes her interactions with Anderson in the following way "... we go over the structure of the class for the upcoming lectures. What are we going to be talking about? Where are we going with the students? What resources are available? ... I would say we work together as colleagues [laughter]. We share good information, ideas - So it's a very collaborative relationship that we have ... I like it." In this quote Maya mentions the shared responsibility and the collaborative aspect of the partnership and clearly sees the value in this type of partnership for the students taking the biochemistry course. Later in the interview, Anderson emphasizes a shared goal of supporting the students and how they collaborate on specific tasks for the class. "There was an example of [when] I didn't have practice problems ... that I liked, and so we actually sat down together- that was like an hour and a half meeting- and trolled through some internet resources and a couple of textbooks and put together a couple advanced problems together." Working together on crafting new practice problems helps situate them within a shared, collaborative, learning community.

Anderson clearly identifies the benefits she receives from the partnership. Specifically, she explains how Maya supports some items that she felt weak in: "[Maya] is more organized than I am and ... has kept me on track ... also the expertise of seeing this material much more recently than I have ... she understands better what it's like to struggle." The excerpts from the interview are consistent with the weekly preparation sessions between Anderson and Maya. During one of the weekly preparation sessions they discussed the material for the upcoming week and looked at what happened in past semesters. They were very detailed in terms of the content and the timing of instruction with both providing their input and ideas. Together, they examined the rough schedule for the semester, and together they decided what content to focus on and what could be left out. Additionally, they discussed an upcoming exam together and how exam questions should be structured.

\section{Challenges and benefits}

Collaborative partnerships can be challenging for faculty because they require a greater investment in time and a willingness to give up some control as their prioritize LAs' expertise as learners. However, these relationships have the potential to positively impact classroom structures as the case of Maya and Anderson illustrates. We also see that listening to LA feedback and ideas can deeply impact the LAs, supporting the work of Close et. al. [4]. One LA 
stated that "... the fact that I'm dealing with people with doctorates, that's not something I normally do ... it gives me a sense of ownership ... knowing that I'm actually becoming a professional myself ... as long as faculty stay open to that opportunity to engage with ... LA students." Importantly, the student indicated that it was not simply their classroom interactions that positively impacted their identity - the rich relationship with a faculty member seeking to listen to them played a significant role.

\section{FROM LOCAL TO NATIONAL PARTNERSHIPS}

While weekly preparation sessions and other elements of the LA Model foster leadership, create space for student voice, and allow rich partnerships to develop at the local level, the LA Alliance creates additional spaces for leadership on a broader scale. Students, as well as others, often benefit from knowing they are part of something bigger, something that extends beyond their respective colleges and universities.

When you visit the LA Alliance webpage you see a membership map of institutions that have LA Programs. The message here is that this is an international network of individuals and institutions that share common goals and can learn from each other. The International LA Workshop, held each fall at the University of Colorado, and the regional workshops, held in the spring at locations around the US all have an LA Panel, where LAs answer questions from faculty, staff, and other students. The panel creates a space for student voice and expertise on a larger scale. One LA commented on his role in the panel mentioning that "... the LA Panel was a good experience ... I was able to ... meet professors ... - they asked intelligent questions that really made me appreciate what I was doing. Initially, I was just doing this ... because this is what I love to do. I realized that it's a big thing that I'm doing and people take it serious and people set up conferences just to interview me." As LAs better understand their roles at the national level, this can then influence their roles and identity at the local level.

\section{CONCLUSIONS}

The LA Model creates unique opportunities for studentfaculty collaboration. In a number of the interviews, LAs mentioned that they have specific ideas about courses and the LA Model that they would like to share with faculty. This requires faculty to be willing to give up certain amounts of control. In both the faculty driven collaboration and the collaborative partnership, LAs can have a large effect on classroom practice when faculty are receptive to feedback, as we see in the partnership between Maya and Anderson. This type of partnership requires faculty to reflect on their own identity and membership in different communities of practice and can be challenging. One faculty member highlighted possible obstacles to collaborative partnerships, stating that "if we want ... to promote this teaching partnership - I don't know how comfortable my colleagues are - I have education background - I'm open to several things - ... I don't know [if others are]." This highlights the importance of creating spaces in our specific LA Programs that provide opportunities for identity change on both the LA side and the faculty side, similar to how the Alliance creates space for LA voice. Viewing the data in the context of learning communities and identity as teachers, professionals, and science people can help us leverage resources and address obstacles that can aid or hinder collaboration in course and program revision. This work also highlights the significant role that weekly preparation sessions play in the LA Model.

\section{AKNOWLEDGEMENTS}

This work is supported by the National Science Foundation (DUE\#1524829), the Department of Education, and the CSU Center for STEM Education and Research. Any opinions, findings, and conclusions expressed in this material are those of the authors and do not necessarily reflect the views of these agencies. This work is Contribution No. LAA-42 of the International Learning Assistant Alliance.
[1] http://laa.colorado.edu/ retrieved 6/25/2016

[2] V. Otero, S. Pollock, and N. Finkelstein, Am. J. Phys., 78, 11 (2010).

[3] K.E. Gray and V. Otero, in AIP Conference Proceedings, edited by C. Henderson, M. Sabella, and L. Hsu, (AIP Publishing, NY, 2008), p. 123.

[4] E.W. Close, J. Conn, and H.G. Close, Physical Review Physics Education Research, 12, 1 (2016).

[5] M.S. Sabella, A.G. Van Duzor, J. Passehl, and K. Weisenburger, The Physics Teacher, 50, 5 (2012).

[6] M.S. Sabella, A.D. Robertson, A.G. Van Duzor, in Effective Practices in Preservice Physics Teacher Education, edited by E. Brewe and C. Sandifer (APS,
College Park, MD, 2015), p. 117

[7] M.S. Sabella and A.G. Van Duzor, in AIP Conference Proceedings (AIP Publishing, NY, 2013), p. 34 .

[8] www.radicalcartography.net/index.html?chicagodots retrieved 6/25/2016

[9] C. Emdin, Online Yearbook of Urban Learning, Teaching, and Research, 46, 52 (2006).

[10] E. Wenger, Communities of practice: Learning, meaning, and identity (Cambridge University Press, Cambridge, UK, 1999)

[11] K. Tobin, Teaching Education, 17, 2, (2006).

[12] C. Emdin, For White Folks Who Teach in the Hood... and the Rest of Y'all Too: Reality Pedagogy and Urban Education (Beacon Press, 2016). 